\title{
WEAK COMMUTATIVITY AND FINITENESS PROPERTIES OF GROUPS
}

\author{
MARTIN R. BRIDSON AND DESSISLAVA H. KOCHLOUKOVA
}

\begin{abstract}
We consider the group $\mathfrak{X}(G)$ obtained from $G * G$ by forcing each element $g$ in the first free factor to commute with the copy of $g$ in the second free factor. Deceptively complicated finitely presented groups arise from this construction: $\mathfrak{X}(G)$ is finitely presented if and only if $G$ is finitely presented, but if $F$ is a non-abelian free group of finite rank then $\mathfrak{X}(F)$ has a subgroup of finite index whose third homology is not finitely generated.
\end{abstract}

\section{INTRODUCTION}

In [19] Saïd Sidki defined a functor $\mathfrak{X}$ that assigns to a group $G$ the group $\mathfrak{X}(G)$ obtained from $G * G$ by forcing each element $g$ in the first free factor to commute with the copy of $g$ in the second free factor. More precisely, taking a second copy $\bar{G}$ of $G$ and fixing an isomorphism $g \mapsto \bar{g}$, one defines $\mathfrak{X}(G)$ to be the quotient of the free product $G * \bar{G}$ by the normal subgroup $\langle\langle[g, \bar{g}]: g \in G\rangle\rangle$. We shall show that this construction is an intriguing new source of finitely presented groups.

At first glance, there seems little reason to believe that $\mathfrak{X}(G)$ can be defined by finitely many of the given relations, even if $G$ is finitely presented, but this is in fact the case:

Theorem A. $\mathfrak{X}(G)$ is finitely presented if and only if $G$ is finitely presented.

We shall derive an explicit finite presentation for $\mathfrak{X}(G)$ from a finite presentation for $G$; see Theorem 1.5. For a free group of rank 2 ,

$$
\mathfrak{X}\left(F_{2}\right)=\left\langle a, b, \bar{a}, \bar{b} \mid[w, \bar{w}], \quad w \in \Upsilon_{2}\right\rangle,
$$

where $\Upsilon_{2}=\left\{a, b, a b, a b^{-1}, a^{-1} b, b a, a b a, a^{-1} b a, a b a^{-1}, b a b, b^{-1} a b, b a b^{-1}\right\}$. For free groups of higher rank, the number of commutators in our presentation of $\mathfrak{X}\left(F_{m}\right)$ grows exponentially with $m$.

It would be quite wrong to interpret Theorem $\AA$ as saying that $\mathfrak{X}(G)$ is a less complicated group than one first imagines. Even for a group as easily understood as a free group $F$, it transpires that $\mathfrak{X}(F)$ is an exotic creature. For example, $\mathfrak{X}(F)$ does not have a classifying space with only finitely many 3 -cells. Recall that a group $G$ is of type $\mathrm{FP}_{n}$ if the trivial $\mathbb{Z} G$-module $\mathbb{Z}$ has a projective resolution that is finitely generated up to dimension $n$. For more details on groups of type $\mathrm{FP}_{n}$ the reader is refered to 4 .

Theorem $\mathbf{B}$. If $F$ is a non-abelian free group, then $\mathfrak{X}(F)$ is not of type $\mathrm{FP}_{3}$.

What we shall actually prove is that $\mathfrak{X}(F)$ has a subgroup of finite index whose third homology $H_{3}(-, \mathbb{Z})$ is not finitely generated - see Theorem 4.1 .

The study of higher finiteness properties of groups began with the work of Serre [17] and Wall 21 in the 1960s, and it has remained a rich and active area of research. The first example of a finitely presented group that is not of type $\mathrm{FP}_{3}$ was constructed by Stallings 20] in 1963. Many groups of this sort are now known, but the functorial nature of the construction in Theorem $B$ is striking.

We also prove a homological version of Theorem A. In the light of Theorem B, one cannot hope to extend this theorem beyond dimension 2 .

2000 Mathematics Subject Classification. Primary 20F05; Secondary 20J05, 20E06.

Key words and phrases. groups, finiteness properties, weak commutativity.

The first author was supported in part by a Wolfson Research Merit Award from the Royal Society. The second author was supported in part by regular grant 2016/05678-3 from FAPESP and "bolsa de produtividade em pesquisa" 303350/2013-0 CNPq, Brazil. 
Theorem C. $\mathfrak{X}(G)$ has homological type $\mathrm{FP}_{2}$ if and only if $G$ has type $\mathrm{FP}_{2}$.

In contrast to Theorem B. Kochloukova and Sidki [13] showed that if $G$ is a soluble group of type $\mathrm{FP}_{\infty}$ then $\mathfrak{X}(G)$ is also soluble group of type $\mathrm{FP}_{\infty}$. The functor $\mathfrak{X}$ preserves many other interesting classes of groups. For example, Sidki [19, Thm. C] showed that if $G$ lies in any of the following classes, then $\mathfrak{X}(G)$ lies in the same class: finite $\pi$-groups, where $\pi$ is a set of primes; finite nilpotent groups; solvable groups. Gupta, Rocco and Sidki [11] proved that the class of finitely generated nilpotent groups is closed under $\mathfrak{X}$, and Lima and Oliveira [15] proved the same for polycyclic-by-finite groups. Kochloukova and Sidki [13] also proved a forerunner of Theorem A: if $G$ is finitely presented and $G^{\prime} / G^{\prime \prime}$ is finitely generated then $\mathfrak{X}(G)$ is finitely presented.

Our original proof of Theorem A relied on the basic structural results for $\mathfrak{X}(G)$ established by Sidki in his seminal paper [19], as well as the VSP criterion for finite presentability established by Bridson, Howie, Miller and Short [7, Thm. A], and a finiteness result concerning the abelianisation of a special subgroup $L(G)<\mathfrak{X}(G)$ that was established by Lima and Oliveira [15]. A shortcoming of this original proof was that it did not give an estimate on the number of relations needed to present $\mathfrak{X}(G)$; it was in seeking to address this that we discovered the proof presented in Section 1. In this approach, a decomposition scheme for planar diagrams leads to an inductive proof that all of the relations $[w, \bar{w}]$ in the definition of $\mathfrak{X}(G)$ follow from a finite set of a particular form. We believe that this technique is of interest in its own right and expect it to find further applications.

In a subsequent paper [8] we shall explore the isoperimetric properties of $\mathfrak{X}(G)$ and prove, among other things, that the class of virtually nilpotent groups is closed under $\mathfrak{X}$.

This paper is organised as follows. In Section 1 we present our diagrammatic proof that $\mathfrak{X}(F)$ is finitely presented if $F$ is a finitely generated free group; the proof yields an explicit finite presentation. Theorem A follows easily from the special case $G=F$. In Section 2 we recall some of Sidki's basic structural results for $\mathfrak{X}(G)$ and its normal subgroups $L=L(G), D=D(G)$ and $W=L \cap D$, and we relate Theorem A to these structures. In Section 3 we prove Theorem C. In Section 4 we prove Theorem B. The proof relies on an analysis of the LHS spectral sequences associated to the maps $\mathfrak{X}(F) \rightarrow \mathfrak{X}(F) / W$ and $L \rightarrow L / W$; results concerning the homology of subdirect products of free groups (à la [6]) play a key role in this analysis.

Acknowledgement: We thank the anonymous referee for their comments and for generously converting our hand-drawn pictures into the diagrams in their current form.

\section{The finite presentability of $\mathfrak{X}(G)$}

Let $F$ be the free group with basis $\left\{a_{1}, \ldots, a_{m}, \bar{a}_{1}, \ldots, \bar{a}_{m}\right\}$. Let $\mathcal{M}$ be the free monoid (set of finite words) with basis the $4 m$-letter alphabet consisting of the symbols $a_{i}, \bar{a}_{i}$ and formal inverses $a_{i}^{-1}, \bar{a}_{i}^{-1}$. Let $F(\mathcal{A})$ (resp. $\mathcal{M}(\mathcal{A})$ ) be the subgroup (resp. submonoid) of $F$ (resp. $\mathcal{M}$ ) generated by $\mathcal{A}=\left\{a_{1}^{ \pm 1}, \ldots, a_{m}^{ \pm 1}\right\}$. We shall reserve the term word for elements of $\mathcal{M}$ (rather than $F$ ) and write $|w|$ to denote the length of a word. We continue to write $w$ when considering the image of $w \in \mathcal{M}$ in $F$ and its quotients.

Given a subset $S \subset F$, we adopt the standard terminology mod $S$ when describing properties of the group $F / N_{S}$, where $N_{S}$ is the normal subgroup generated by $S$. Thus, for example, $w=1$ mod $S$ means that the image of $w$ in $F / N_{S}$ is trivial. We write $\bar{w}$ for the word obtained from $w \in \mathcal{M}(\mathcal{A})$ by replacing each $a_{i}^{ \pm 1}$ with $\bar{a}_{i}^{ \pm 1}$

We shall be particularly concerned with words of the form

$$
\square_{w}:=w^{-1} \bar{w}^{-1} w \bar{w}
$$

for $w \in \mathcal{M}(\mathcal{A})$. In $F$ we have $\square_{w}=[w, \bar{w}]$. Define

$$
\square((n))=\left\{\left[w, \bar{w}^{-1}\right]|| w \mid \leq n\right\} .
$$

Theorem $\AA$ is equivalent to the assertion that there exists an $n_{0}$ such that $\square_{u}=1 \bmod \square\left(\left(n_{0}\right)\right)$ for all $u \in \mathcal{M}(\mathcal{A})$. Thus our objective is to find equations in $F$ that exhibit the triviality of $\square_{w}$ in $\mathfrak{X}(F)$ as a consequence of shorter relations. 
1.1. Identities from diagrams. The use of planar diagrams to explore relations in groups is well established. Most applications can be interpreted as forms of van Kampen's Lemma, and this applies to the following simple observation.

Lemma 1.1. Let $X$ be a finite, connected, planar graph with edges labelled by elements of the free group $F$. Let $r_{1}, \ldots, r_{m}$ be the elements labelling the boundary cycles of the compact connected components of $\mathbb{R}^{2} \backslash X$ and let $r_{0}$ be the label on the boundary cycle of the infinite component. (These labels can be read from any point on the cycle, proceeding with either orientation.) For all $i, j \in\left\{r_{0}, \ldots, r_{m}\right\}$, let $R_{i j}=\left\{r_{k} \mid k \neq i, j\right\}$. Then,

$$
r_{i}=1 \bmod R_{i j} \Longleftrightarrow r_{j}=1 \bmod R_{i j} \text {. }
$$

A topological explanation for this fact is the following: consider the 1-vertex graph with directed edges labelled by the elements of a fixed generating set for $F$ and attach $m-1$ discs to this along the loops labelled $r_{k} \in R_{i j}$. The fundamental group of the resulting 2-complex is $F /\left\langle\left\langle R_{i j}\right\rangle\right\rangle$ and the loop labelled $r_{i}$ is freely homotopic to that labelled $r_{j}^{ \pm 1}$ (with the sign determined by the original choices of orientation on the boundary loops for $X$ ).

An algebraic proof can be obtained by following the standard proof of van Kampen's lemma. We illustrate this with the two special cases that are central to our proof of Theorem A.

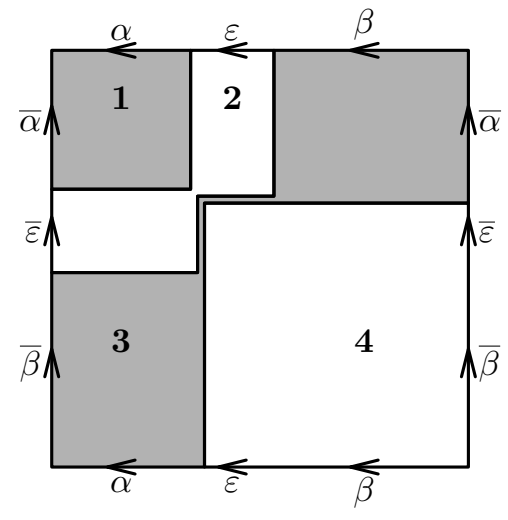

(i)

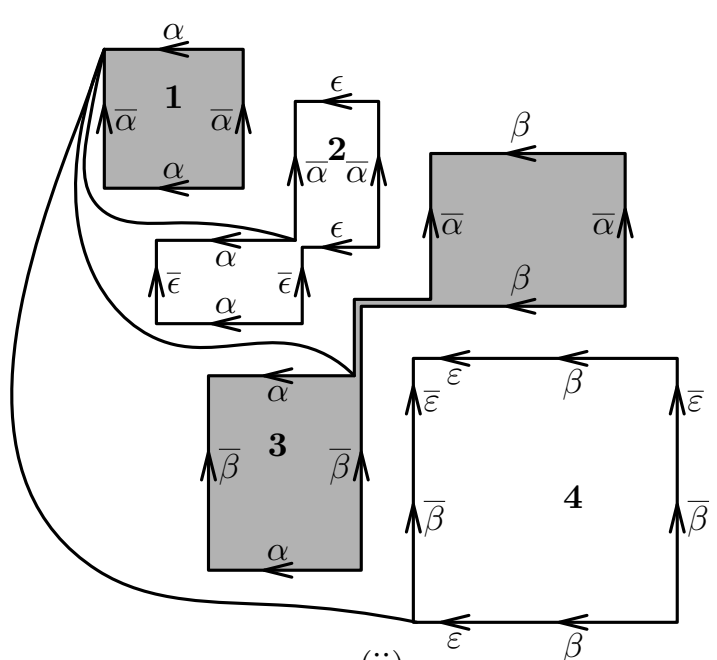

(ii)

FigURE 1. diagram with boundary $\square_{\beta \varepsilon \alpha}=1$

In this diagram $\alpha, \varepsilon, \beta$ are arbitrary words and all segments in the interior are labelled with the words labelling the segments of the boundary that are vertical or horizontal translates of them. We shall read all labels by proceeding clockwise around boundary faces.

We perform algebraic manipulations in the free group, guided by reading the outer boundary of the decomposition shown in fig 1(ii). The validity of these manipulations can be verified 11 by freely reducing the righthand side of equation (1.1); the four bracketed factors correspond to the four faces of the diagram.

Our notational conventions are $[x, y]:=x^{-1} y^{-1} x y$ and $x^{y}:=y^{-1} x y$.

$$
[\beta \varepsilon \alpha, \bar{\beta} \overline{\varepsilon \alpha}]=[\alpha, \bar{\alpha}]\left(\left[\bar{\alpha}^{-1}, \varepsilon\right]\left[\alpha^{-1}, \bar{\varepsilon}\right]^{-1}\right)^{\alpha \bar{\alpha}}\left(\left[\bar{\alpha}^{-1}, \beta\right]^{\varepsilon^{-1}}\left[\bar{\beta}, \alpha^{-1}\right]\right)^{\alpha \overline{\varepsilon \alpha}}\left[(\bar{\beta} \bar{\varepsilon})^{-1}, \beta \varepsilon\right]^{\alpha \bar{\beta} \bar{\varepsilon}} \text {. }
$$

When $\varepsilon$ is the empty word, figure 1 simplifies to figure 2 and equation (1.1) reduces to:

$$
[\beta \alpha, \bar{\beta} \bar{\alpha}]=[\alpha, \bar{\alpha}]\left(\left[\bar{\alpha}^{-1}, \beta\right]\left[\bar{\beta}, \alpha^{-1}\right]\right)^{\alpha \bar{\alpha}}\left[\bar{\beta}^{-1}, \beta\right]^{\alpha \bar{\beta} \bar{\alpha}} .
$$

Lemma 1.2. For all non-empty words $\alpha, \varepsilon, \beta \in \mathcal{M}(\mathcal{A})$ with $|\alpha|+|\varepsilon|+|\beta|=n+1$,

$$
\square_{\beta \varepsilon \alpha}=1 \bmod \square((n)) \Longleftrightarrow\left[\left[\bar{\alpha}^{-1}, \beta\right], \varepsilon \bar{\varepsilon}^{-1}\right]=1 \bmod \square((n)) .
$$

\footnotetext{
${ }^{1}$ discovering the decomposition algebraically is harder than verifying it!
} 

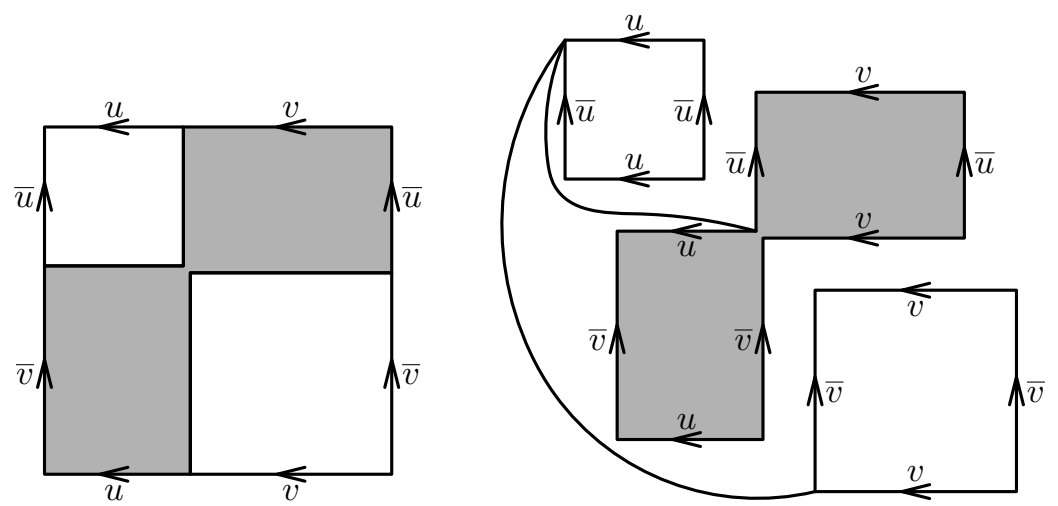

Figure 2. $\left[\bar{u}^{-1}, v\right]\left[\bar{v}, u^{-1}\right]=1$

Proof. Equation (1.2) shows that for all words $u, v \in \mathcal{M}(\mathcal{A})$ with $|u|+|v| \leq n$ we have

$$
\left[\bar{u}^{-1}, v\right]\left[\bar{v}, u^{-1}\right]=1 \bmod \square((n)) .
$$

Thus, mod $\square((n))$, each factor on the right hand side of (1.1) is trivial, except possibly the third, and for that we have,

$$
\left[\bar{\alpha}^{-1}, \beta\right]^{\varepsilon \bar{\varepsilon}^{-1}}\left[\bar{\beta}, \alpha^{-1}\right]=1 \Longleftrightarrow\left[\bar{\alpha}^{-1}, \beta\right]^{\varepsilon^{-1}}=\left[\bar{\beta}, \alpha^{-1}\right]^{-1}=\left[\bar{\alpha}^{-1}, \beta\right],
$$

where the last equality is an application of (1.3).

Lemma 1.3. For all non-empty words $\alpha, \varepsilon, \beta \in \mathcal{M}(\mathcal{A})$ with $|\alpha|+|\varepsilon|+|\beta|=n+1$,

$$
\square_{\beta \varepsilon \alpha}=1 \bmod \square((n)) \Longleftrightarrow \square_{\beta \varepsilon^{-1} \alpha}=1 \bmod \square((n)) .
$$

Proof. The second of the equivalences in the following chain is justified because the identity $\bar{\varepsilon} \varepsilon^{-1}=\varepsilon^{-1} \bar{\varepsilon}\left[\bar{\varepsilon}, \varepsilon^{-1}\right]$ implies $\left(\varepsilon \bar{\varepsilon}^{-1}\right)^{-1}=\varepsilon^{-1} \bar{\varepsilon} \bmod \square((n-1))$. The other equivalences are instances of Lemma 1.2. All calculations are $\bmod \square((n))$.

$$
\square_{\beta \varepsilon \alpha}=1 \Longleftrightarrow\left[\left[\bar{\alpha}^{-1}, \beta\right], \varepsilon \bar{\varepsilon}^{-1}\right]=1 \Longleftrightarrow\left[\left[\bar{\alpha}^{-1}, \beta\right], \varepsilon^{-1} \bar{\varepsilon}\right]=1 \Longleftrightarrow \square_{\beta \varepsilon^{-1} \alpha}=1 .
$$

1.2. A reduction scheme. We maintain the notation established at the beginning of this section; in particular $F$ is the free group of rank $m$. The following proposition shows that in order to present $\mathfrak{X}(F)$ one only needs to impose the relations $\square_{u}$ with $|u| \leq m+1$.

Proposition 1.4. For all $w \in \mathcal{M}(\mathcal{A})$, if $|w| \geq m+2$ then $\square_{w}=1 \bmod \square((m+1))$.

Proof. It is enough to prove that $\square_{w}=1 \bmod \square((|w|-1))$ if $|w| \geq m+2$.

Consider a word $w=\beta_{0} w_{0} \alpha_{0}$ of length $n+1 \geq m+2$ with $\alpha_{0}, w_{0}, \beta_{0} \in \mathcal{M}(\mathcal{A})$ and $\left|\alpha_{0}\right|=\left|\beta_{0}\right|=1$. By making repeated use of Lemma 1.3, we can permute the letters of $w_{0}$

$$
\ldots a b \ldots \sim \ldots(a b)^{-1} \ldots=\ldots b^{-1} a^{-1} \ldots \sim \ldots b a \ldots,
$$

and thus, after free reduction, transform $w_{0}$ into a word of the form $w_{0}^{\prime}=a_{i_{1}}^{p_{1}} \ldots a_{i_{s}}^{p_{s}}$ with $\left|w_{0}^{\prime}\right| \leq\left|w_{0}\right|$ and $1 \leq i_{1}<i_{2}<\cdots<i_{s} \leq m$, such that

$$
\square_{w}=1 \bmod \square((n)) \Longleftrightarrow \square_{\beta_{0} w_{0}^{\prime} \alpha_{0}}=1 \bmod \square((n)) .
$$

Using Lemma 1.3 again, we can assume $p_{i}>0$. And by transforming subwords $a a$ into $a a^{-1}$ and cancelling, we can actually assume that $w_{0}^{\prime}$ is a monomial word, i.e. each $p_{i}=1$. When modified in this way, $\left|w_{0}^{\prime}\right| \leq m$. Next, if $\alpha_{0}=a_{k}^{ \pm 1}$ for some $k \in\left\{i_{1}, \ldots, i_{s}\right\}$, then we can permute $a_{k}$ to the end of $w_{0}^{\prime}$ and cancel it with $\alpha_{0}$ (using Lemma 1.3 to replace it by its inverse if necessary). Thus we can assume that $k \notin\left\{i_{1}, \ldots, i_{s}\right\}$. Similarly, $\beta_{0}=a_{j}^{ \pm 1}$ with $j \notin\left\{i_{1}, \ldots, i_{s}\right\}$ (but it is possible that $\left.a_{j}=a_{k}\right)$. With these final assumptions, we have $\left|w_{0}^{\prime}\right| \leq m-1$, so from (1.4) we have $\square_{w}=1$ $\bmod \square((n))$, provided $n \geq m+1$. 
1.3. A presentation of $\mathfrak{X}(F)$. First we consider the case where $F=F_{2}$ is free of rank 2 with basis $\{a, b\}$. The proof of Proposition 1.4 shows that in order to present $\mathfrak{X}\left(F_{2}\right)$ it is sufficient to impose 12 relations of the form $\square_{w}=1$. To obtain the list of sufficient relations, one begins with all words of length at most 2 and adds to it those of length 3 obtained by following the proof of Proposition 1.4 with $w_{0}^{\prime}=a$ and $w_{0}^{\prime}=b$. The list can then be shortened by noting that $\square_{w}=1$ implies $\square_{w^{-1}}=1$.

$$
\left.\mathfrak{X}\left(F_{2}\right)=\langle a, b, \bar{a}, \bar{b}|[w, \bar{w}] \text { if } w \in \Upsilon_{2}\right\rangle,
$$

where $\Upsilon_{2}=\left\{a, b, a b, a b^{-1}, a^{-1} b, b a, a b a, a^{-1} b a, a b a^{-1}, b a b, b^{-1} a b, b a b^{-1}\right\}$.

Consider now the case where $F=F_{3}$ is free of rank 3 with basis $\{a, b, c\}$. In this case, we include 3 words of length 1 and 4 words of length 2 for each pair of distinct basis elements. To these 15 words we add further relations accounting for each of the possibilities for $w_{0}^{\prime}$ in the proof of Proposition 1.4 (in its final, positive, square-free form). The possibilities are

$$
a, b, c, a b, b c, a c .
$$

In each case we have to account for the different initial and terminal letters $\alpha_{0}$ and $\beta_{0}$ (with signs). If $w_{0}^{\prime}=a$, there are 10 possibilities (after removing redundancies such as $b a c \sim(b a c)^{-1} \sim$ $c^{-1} a b^{-1}$ by appealing to Lemma 1.3 ):

$$
b a b, b a b^{-1}, b^{-1} a b, b a c, b a c^{-1}, b^{-1} a c, c a b, c a c, c a c^{-1}, c^{-1} a c .
$$

Likewise we add 10 words for $w_{0}^{\prime}=b$ and 10 for $w_{0}^{\prime}=c$.

For $w_{0}^{\prime}=a b$, the relations that we add are $\square_{w}$ with $w \in\left\{c a b c, c a b c^{-1}, c^{-1} a b c\right\}$. And similarly for $w_{0}^{\prime} \in\{b c, a c\}$.

Thus we obtain a presentation of $\mathfrak{X}\left(F_{3}\right)$ with generators $\{a, b, c, \bar{a}, \bar{b}, \bar{c}\}$ subject to 54 relations of the form $[w, \bar{w}]=1$, with $|w| \leq 4$.

In the general case, where $F_{m}$ is free of rank $m$, say with a basis $a_{1}, \ldots, a_{m}$, our algorithm yields $m$ words of length 1 together with $2 m(m-1)$ words of length 2 and, for each $s<m$ and each of the $\left(\begin{array}{c}m \\ s\end{array}\right)$ monomials $w_{0}=a_{i_{1}} \ldots a_{i_{s}}$ with $1 \leq i_{1}<\cdots<i_{s} \leq m$, an additional $3(m-s)$ relations of the form $a_{k} w_{0} a_{k}, a_{k} w_{0} a_{k}^{-1}, a_{k}^{-1} w_{0} a_{k}$ where $k \in\{1, \ldots, m\} \backslash\left\{i_{1}, \ldots, i_{s}\right\}$ and $4\left(\begin{array}{c}m-s \\ 2\end{array}\right)$ relations of the form $a_{k} w_{0} a_{j}, a_{k} w_{0} a_{j}^{-1}, a_{k}^{-1} w_{0} a_{j}, a_{j} w_{0} a_{k}$ where $k \neq j$ and $k, j \in\{1, \ldots, m\} \backslash\left\{i_{1}, \ldots, i_{s}\right\}$. Thus $\mathfrak{X}\left(F_{m}\right)$ has a presentation with $2 m$ generators and

$$
v_{m}=m+2 m(m-1)+\sum_{s=1}^{m-1}\left(\begin{array}{c}
m \\
s
\end{array}\right)\left[3(m-s)+4\left(\begin{array}{c}
m-s \\
2
\end{array}\right)\right]
$$

relations of the form $[w, \bar{w}]=1$, with $|w| \leq m+1$. Note that as $m$ increases, this number of relations grows exponentially.

A somewhat less economical presentation is given by the following distillation of the proof of Proposition 1.4.

\subsection{A finite presentation of $\mathfrak{X}(G)$.}

Theorem 1.5. Let $G=\left\langle a_{1}, \ldots, a_{m} \mid r_{1}, \ldots, r_{n}\right\rangle$ be a finitely presented group. Then

$$
\left.\mathfrak{X}(G)=\left\langle a_{1}, \ldots, a_{m}, \bar{a}_{1}, \ldots, \bar{a}_{m}\right| r_{1}, \ldots, r_{n}, \bar{r}_{1}, \ldots, \bar{r}_{n},[w, \bar{w}] \text { if } w \in \Upsilon_{m}\right\rangle,
$$

where $\Upsilon_{m}$ consists of the elements of $\left\{a_{i}, a_{i} a_{j}, a_{i} a_{j}^{-1}, a_{i}^{-1} a_{j}, a_{j} a_{i}: 1 \leq i<j \leq n\right\}$ together with words of the form $w=\beta_{0} w_{0} \alpha_{0}$ where $w_{0}$ is a monomial word $a_{i_{1}} \ldots a_{i_{s}}$ with $1 \leq i_{1}<\cdots<i_{s} \leq m$, and $\alpha_{0}=a_{j}^{ \pm 1}$ and $\beta_{0}=a_{k}^{ \pm 1}$ with $j, k \notin\left\{i_{1}, \ldots, i_{s}\right\}$.

Proof. It follows immediately from the definition that $\mathfrak{X}(G)$ is the quotient of $\mathfrak{X}\left(F_{m}\right)$ by the normal subgroup generated by the $r_{i}$ and $\bar{r}_{i}$, and the presentation of $\mathfrak{X}\left(F_{m}\right)$ comes from the proof of Proposition 1.4. 


\section{On the Structure of the Group $\mathfrak{X}(G)$}

Following Sidki [19], we highlight some subgroups and decompositions of $\mathfrak{X}(G)$. We follow the notations from [19] except that we write $\bar{G}$ and $\bar{g}$ where Sidki writes $G^{\psi}$ and $g^{\psi}$.

By means of careful commutator calculations, Sidki proves that the following subgroups of $\mathfrak{X}(G)$ are normal [19, Lemma 4.1.3]:

$$
D=D(G):=[G, \bar{G}] ; \quad L=L(G):=\left\langle g^{-1} \bar{g} \mid g \in G\right\rangle .
$$

It follows that $D$ is the kernel of the natural map $\mathfrak{X}(G) \rightarrow G \times \bar{G}$ and that $L$ is the kernel of the map $\mathfrak{X}(G) \rightarrow G$ that sends both $g$ and $\bar{g}$ to $g$. This last map has an obvious splitting, giving

$$
\mathfrak{X}(G)=L \rtimes G .
$$

By combining the maps with kernel $D$ and $L$, we obtain a homomorphism

$$
\rho: \mathfrak{X}(G) \rightarrow G \times \bar{G} \times G \cong G \times G \times G
$$

with

The kernel of $\rho$ is

$$
\rho(g)=(g, g, 1), \quad \rho(\bar{g})=(1, g, g) \text { for all } g \in G \text {. }
$$

$$
W=W(G):=D \cap L .
$$

By means of further commutator calculations, Sidki [19, Lemma 4.1.6 (ii)] showed that $D$ commutes with $L$ and therefore $W$ is central in $D L$ (cf. Lemma 1.2 above); in particular, $W$ is abelian.

The image of $\rho$ is

$$
Q_{G}:=\operatorname{im}(\rho)=\left\{\left(g_{1}, g_{2}, g_{3}\right) \mid g_{1} g_{2}^{-1} g_{3} \in[G, G]\right\} .
$$

Note that $\operatorname{im}(\rho)$ contains the commutator subgroup of $G \times G \times G$; $\operatorname{im}(\rho)$ is normal in $G \times G \times G$ with quotient $H_{1}(G, \mathbb{Z})$. Moreover, its projection onto each pair of coordinates is onto, so by the Virtual Surjection to Pairs Theorem [7], if $G$ is finitely presented then $\operatorname{im}(\rho)$ is finitely presented. Thus, when $G$ is finitely presented we have an exact sequence

$$
1 \rightarrow W \rightarrow \mathfrak{X}(G) \rightarrow Q_{G} \rightarrow 1
$$

with $Q_{G}$ finitely presented and $W$ abelian.

The group $\rho(L)$ is normal in $G \times 1 \times G \cong G \times G$; the intersection with each direct factor is the commutator subgroup and the quotient is $H_{1}(G, \mathbb{Z})$. If $G$ is finitely generated then $\rho(L)$ is finitely generated (see [10], for example), but if $G=F$ is a non-abelian free group then $H_{2}(\rho(L), \mathbb{Z})$ is not finitely generated. Thus, in the case $G=F$, we have a central extension

$$
1 \rightarrow W \rightarrow L \rightarrow \rho(L) \rightarrow 1,
$$

where $\rho(L)$ is finitely generated but not finitely presented.

2.1. Finite generation for $L$. We noted above that $\mathfrak{X}(G)=L \rtimes G$. So if $\mathfrak{X}(G)$ is finitely generated then $G$, as a retract, is also finitely generated. Our initial attempts to prove Theorem A focused on a partial converse to this observation, the relevance of which is explained by the following lemma.

Lemma 2.1. Let $F$ be a finitely generated free group. If $\mathfrak{X}(F)$ is finitely presented then $L=$ $L(F)$ is finitely generated.

Proof. Let $\pi: \mathfrak{X}(F)=L \rtimes F \rightarrow F$ be the retraction with kernel $L$ and recall that $[L, D]=1$. For a fixed $g_{0} \in D \backslash L$ consider $\pi\left(g_{0}\right) \in F$. Marshall Hall's theorem [12] provides a subgroup $F_{0}<F$ of finite index such that $\pi\left(g_{0}\right)$ is a primitive element of $F_{0}$. Consider $G_{0}=\pi^{-1}\left(F_{0}\right)=L \rtimes F_{0}$. As a subgroup of finite index in $\mathfrak{X}(F)$, this is finitely presented. By choosing a different section of $\pi$ if necessary, we may assume that $g_{0} \in \tilde{F}_{0}=1 \times F_{0}$. Since $\pi\left(g_{0}\right)$ is primitive in $F_{0}$, we know that $g_{0}$ is primitive in $\tilde{F}_{0}$, so $\tilde{F}_{0}=K * F_{1}$ where $K$ is the cyclic subgroup of $\tilde{F}_{0}$ generated by $g_{0}$. Thus $G_{0}$ decomposes as an HNN extension with stable letter $g_{0}$ and relative presentation

$$
G_{0}=\left\langle L \rtimes F_{1}, g_{0} \mid\left[L, g_{0}\right]=1\right\rangle .
$$


Crucially, $G_{0}$ retracts onto $L \rtimes F_{1}$ by the map that sends $g_{0}$ to 1 . Hence $L \rtimes F_{1}$ is finitely presented. By a result of Miller [16, Lemma 2.1], if an HNN extension is finitely presented and the base group is finitely presented, then the associated subgroup must be finitely generated. Thus $L$ is finitely generated.

With Theorem A in hand, this lemma tells us that $L$ is finitely generated, but one can prove this more directly using the following lemma.

Lemma 2.2. Let $\Gamma$ be a group and suppose that $C<\Gamma$ is central. If $\Gamma / C$ and $H_{1}(\Gamma, \mathbb{Z})$ are finitely generated, then $\Gamma$ is finitely generated.

Proof. Let $S \subset \Gamma$ be a finite set whose image generates $\Gamma / C$ and let $\Sigma<\Gamma$ be the subgroup generated by $S$. Each $\gamma \in \Gamma$ can be written in the form $\gamma=z \sigma$ with $z \in C$ and $\sigma \in \Sigma$. For all $x \in \Sigma$ we have $x^{\gamma}=x^{\sigma} \in \Sigma$. Thus $\Sigma$ is normal in $\Gamma$. The central subgroup $C<\Gamma$ maps onto $\Gamma / \Sigma$, which is therefore abelian. So if the images of $c_{1}, \ldots, c_{n} \in \Gamma$ generate $H_{1}(\Gamma, \mathbb{Z})$, then $S \cup\left\{c_{1}, \ldots, c_{n}\right\}$ generates $\Gamma$.

We can apply this lemma in the setting of (2.1) because Lima and Oliveira [15] proved that $H_{1}(L, \mathbb{Z})$ is finitely generated whenever $G$ is finitely generated. We recall their construction.

They consider the wreath product $H=\mathbb{Z} \imath G$, viewing $\oplus_{g \in G} \mathbb{Z}$ as the additive group of the integral group ring $\mathbb{Z} G$; so $H=\mathbb{Z} G \rtimes G$. Focusing on the augmentation ideal $A(G)$ of $\mathbb{Z} G$ and the additive group $I_{2}(G)$ of the ideal of $\mathbb{Z} G$ generated by $\left\{(1-g)^{2} \mid g \in G\right\}$, they prove that the homomorphism of groups $\nu: \mathfrak{X}(G) \rightarrow H / I_{2}(G)$ defined 2 by $\nu(g)=(1, g)$ and $\nu(\bar{g})=(1, g)^{(1,1)}$ restricts to an epimorphism from $L=L(G)$ to $A(G) / I_{2}(G)$ with kernel $[L, L]$. Thus the abelianisation of $L$ is a subgroup of $M=\mathbb{Z} G / I_{2}(G)$. Lima and Oliveira show in [15 that if $G$ is generated by $a_{1}, \ldots, a_{m}$, then $M$ is generated as an abelian group by 1 and the finitely many monomials $a_{i_{1}} \ldots a_{i_{s}}$ with $1 \leq i_{1}<\cdots<i_{j} \leq m$.

Thus we see the monomials that played a crucial role in our proof of Theorem A appear from an alternative perspective.

Moving beyond the case $G=F$, we record:

Proposition 2.3. For all finitely generated groups $G$, the group $L(G)$ is finitely generated.

Proof. The natural map $\mathfrak{X}(F) \rightarrow \mathfrak{X}(G)$ sends $L(F)$ onto $L(G)$ and therefore the latter is finitely generated.

By following the constructions given above, one can give an explicit generating set for $L(G)$. We illustrate this in the 2-generator case, for which we need the following lemma.

Lemma 2.4. If $G$ is generated by $\{a, b\}$, then $P=\left\langle\left(g, g^{-1}\right): g \in G\right\rangle\langle G \times G$ is generated by $\left\{\left(a, a^{-1}\right),\left(b, b^{-1}\right),\left(a b,(a b)^{-1}\right)\right\}$.

Proof. $P$ is the kernel of the map $G \times G \rightarrow H_{1}(G, \mathbb{Z})$ that restricts to the canonical surjection on each factor. It is clear that this is generated by $\left\{\alpha=\left(a, a^{-1}\right), \beta=\left(b, b^{-1}\right)\right\}$ and the normal closure of $c=([a, b], 1)$ (with $\left(1,\left[b^{-1}, a^{-1}\right]\right)$ obtained as $\left.c[\alpha, \beta]^{-1}\right)$, and one can obtain any conjugate of $c$ by noting that $c^{(a, 1)}=c^{\alpha}$ and $c^{(b, 1)}=c^{\beta}$. Thus $P$ is generated by $\{\alpha, \beta, c\}$. And since

$$
([a, b], 1)=\left(a^{-1}, a\right)\left(b^{-1}, b\right)\left(a b,(a b)^{-1}\right),
$$

$P$ is also generated by $\left\{\left(a, a^{-1}\right),\left(b, b^{-1}\right),\left(a b,(a b)^{-1}\right)\right\}$.

Proposition 2.5. If $F$ is the free group with basis $\{a, b\}$, then $L(F)<\mathfrak{X}(F)$ is generated by $\left\{a \bar{a}^{-1}, b \bar{b}^{-1}, a b(\bar{a} \bar{b})^{-1}\right\}$.

Proof. Applying the proof of Lemma 2.2 to (2.1), we see that it is enough to prove that the image of the given subset of $L$ generates both $\rho(L)$ and $H_{1}(L, \mathbb{Z})$. For $\rho(L)$, this is covered by Lemma 2.4.

Lima and Oliveira show in 15 that $M=\mathbb{Z} F / I_{2}(F)$ is generated as an abelian group by $X=\{1, a, b, a b\}$. The augmentation map $\mathbb{Z} F \rightarrow \mathbb{Z}$ (defined by $w \mapsto 1$ for all $w \in F$ ) descends

\footnotetext{
2 note that $(0,1) \in H$ is the identity element, not $(1,1)$
} 
to an epimorphism $\mu: M \rightarrow \mathbb{Z}$ defined by $\mu(x)=1$ (the generator of $\mathbb{Z}$ ) for each $x \in X$. Hence ker $\mu=A(F) / I_{2}(F)$ is generated as an abelian group by $\{1-a, 1-b, 1-a b\}$. And $\nu\left(u \bar{u}^{-1}\right)=1-u$ defines an epimorphism $L \rightarrow A(F) / I_{2}(F)$ with kernel $[L, L]$.

Corollary 2.6. $H_{1}\left(L\left(F_{2}\right), \mathbb{Z}\right) \cong \mathbb{Z}^{3}$.

Proof. $L$ is 3-generator and maps onto $H_{1}(\rho(L), \mathbb{Z})$, which a calculation with the 5 -term exact sequence shows to be $\mathbb{Z}^{3}$; see [10, Theorem A].

\section{Theorem B: Sidki's functor $\mathfrak{X}$ preserves $\mathrm{FP}_{2}$}

We remind the reader that a group $G$ is of type $\mathrm{FP}_{2}$ if there is a projective resolution of the trivial $\mathbb{Z} G$-module $\mathbb{Z}$ that is finitely generated up to dimension 2 . If one expresses $G$ as the quotient of a finitely generated free group $G=F / R$ and considers $M=R /[R, R]$ as a $\mathbb{Z} F$-module (or $\mathbb{Z} G$-module) via the action of $F$ on $R$ by conjugation, then $G$ is of type $\mathrm{FP}_{2}$ if and only if $M$ is finitely generated as a module.

For completeness, we prove the following well known fact; the forward direction is [5, Lemma 2.1].

Lemma 3.1. A group $G$ is of type $\mathrm{FP}_{2}$ if and only if there is a finitely presented group $H$ and an epimorphism $H \rightarrow G$ with perfect kernel.

Proof. First assume that $G$ is of type $\mathrm{FP}_{2}$ and write $G=F / R$ where $F$ is free of finite rank and $R /[R, R]$ is generated as a $\mathbb{Z} F$ module by the image of a finite set $S=\left\{r_{1}, \ldots, r_{m}\right\} \subset R$. In $F$, every element of $R$ can be expressed as a product

$$
r=\rho \prod_{i=1}^{n} \theta_{i} r_{j(i)}^{ \pm 1} \theta_{i}^{-1},
$$

where $\rho \in[R, R]$ and $\theta_{i} \in F$.

Let $N \triangleleft F$ be normal closure of $S$ in $F$, let $H=F / N$ and consider the natural surjection $\pi: H \rightarrow G$. For each $r N \in R / N=\operatorname{ker} \pi$, the equation above projects to an equality $r N=\rho N$ in $H$, and hence $\operatorname{ker} \pi=R / N=[R, R] / N$ is perfect.

Conversely, if $H=F / N$ is finitely presented, with $N=\left\langle\left\langle r_{1}, \ldots, r_{m}\right\rangle\right\rangle$ say, and $H \rightarrow G$ is a surjection with perfect kernel $R / N$, then by hypothesis every $r \in R$ lies in $[R, R] N$. Thus we can express $r$ in the free group as a product of the above form. This shows that $M=R /[R, R]$ is generated as a $\mathbb{Z} F$ module by the images of $\left\{r_{1}, \ldots, r_{m}\right\}$. And as $M$ is the relation module for $G=F / R$, this implies that $G$ is of type $\mathrm{FP}_{2}$.

Theorem 3.2. $G$ is $\mathrm{FP}_{2}$ if and only if $\mathfrak{X}(G)$ is $\mathrm{FP}_{2}$.

Proof. Every retract of a group of type $\mathrm{FP}_{m}$ is $\mathrm{FP}_{m}$, so if $\mathfrak{X}(G)=L \rtimes G$ is $\mathrm{FP}_{m}$ (for any $m$ ) then so is $G$.

Conversely, if $G$ is $\mathrm{FP}_{2}$ then by the lemma there is an epimorphism $\pi: H \rightarrow G$ with kernel $K$ where $H$ is finitely presented and $K$ is perfect. The product of perfect subgroups is perfect and the normal closure of a perfect subgroup is perfect, so the normal subgroup $N<\mathfrak{X}(H)$ generated by $K \cup \bar{K} \subset H \cup \bar{H}$ is perfect. $N$ is the kernel of the map $\pi: \mathfrak{X}(H) \rightarrow \mathfrak{X}(G)$ defined by $h \mapsto \pi(h)$ and $\bar{h} \mapsto \overline{\pi(h)}$. From Theorem A we know that $\mathfrak{X}(H)$ is finitely presented, so $\mathfrak{X}(G)$ is of type $\mathrm{FP}_{2}$, by Lemma 3.1 .

In 3. Bestvina and Brady constructed groups that are of type $\mathrm{FP}_{\infty}$ but are not finitely presented. Recently, Leary [14] showed that there are uncountably many groups with these properties.

Corollary 3.3. If $G$ is a group of type $\mathrm{FP}_{2}$ that is not finitely presented, then $\mathfrak{X}(G)$ is a group of type $\mathrm{FP}_{2}$ that is not finitely presented.

\section{4. $\mathfrak{X}(F)$ IS NOT OF TYPE $\mathrm{FP}_{3}$}

We prove the following strengthening of Theorem B. 
Theorem 4.1. If $F$ is a free group of finite rank $m \geq 2$, then there is a subgroup of finite index $\Gamma<\mathfrak{X}(F)$ with $H_{3}(\Gamma, \mathbb{Z})$ not finitely generated.

We assume that the reader is familiar with the Lyndon-Hochschild-Serre (LHS) spectral sequence associated to a short exact sequence of groups.

Lemma 4.2. Let $F$ be a free group of finite rank $m \geq 2$. If $H_{3}(\Gamma, \mathbb{Z})$ is finitely generated for all subgroups of finite index $\Gamma \leq \mathfrak{X}(F)$, then $W(F)$ is not finitely generated.

Proof. $W=W(F)$ is the kernel of the natural map $\rho: \mathfrak{X}(F) \rightarrow F \times F \times F$ described in Section 2, the image of which is a finitely presented, full subdirect product of infinite index. Every such subgroup of $F \times F \times F$ is finitely presented but has a subgroup of finite index $\Delta$ with $H_{3}(\Delta, \mathbb{Z})$ not finitely generated [6, Thm. C]. If the abelian group $W$ were finitely generated, then it would be of type $\mathrm{FP}_{\infty}$, and from the LHS spectral sequence $E_{p, q}^{2}=H_{p}\left(\Delta, H_{q}(W, \mathbb{Z})\right)$ that converges to $H_{p+q}\left(\rho^{-1}(\Delta), \mathbb{Z}\right)$, we would conclude that $H_{3}\left(\rho^{-1}(\Delta), \mathbb{Z}\right)$ was not finitely generated, contrary to hypothesis.

In more detail, $E_{3,0}^{\infty}$ is obtained from $E_{3,0}^{2}=H_{3}(\Delta, \mathbb{Z})$ by passing to the kernel of $d_{2}: E_{3,0}^{2} \rightarrow$ $E_{1,1}^{2}=H_{1}(\Delta, W)$ and then the kernel of $d_{3}: E_{3,0}^{3} \rightarrow E_{0,2}^{3}$, whose codomain is a quotient of $H_{2}(W, \mathbb{Z})$; if $W$ were finitely generated then $H_{1}(\Delta, W)$ would be as well (since $\Delta$ is finitely genereated), as would $H_{2}(W, \mathbb{Z})$; but this would imply that $E_{3,0}^{\infty}$ (whence $H_{3}\left(\rho^{-1}(\Delta), \mathbb{Z}\right)$ ) was not finitely generated.

Lemma 4.3. Let $1 \rightarrow A \rightarrow G \rightarrow Q \rightarrow 1$ be a central extension of a finitely generated group $Q$ of cohomological dimension $\operatorname{cd}(Q) \leq 2$. If the abelianisation of $Q$ is infinite and $A$ is not finitely generated, then $\mathrm{H}_{2}(G, \mathbb{Z})$ is not finitely generated.

Proof. As $\operatorname{cd}(Q) \leq 2$, all of the non-zero terms on the $E^{2}$ page of the LHS spectral sequence in homology associated to the given extension are concentrated in the first three columns. Thus $E^{3}=E^{\infty}$ and the $d_{2}$ differentials beginning and ending at $E_{1,1}^{2}=H_{1}(Q, A)$ are both zero maps. So $E_{1,1}^{\infty}$, which is a section of $H_{2}(G, \mathbb{Z})$, is $H_{1}(Q, A) \cong H_{1}(Q, \mathbb{Z}) \otimes A$, which contains a copy of $A$, since $H_{1}(Q, \mathbb{Z})$ has $\mathbb{Z}$ as a direct summand. As $A$ is not finitely generated, neither is $\mathrm{H}_{2}(G, \mathbb{Z})$.

We shall need the following homological variation of Miller's lemma [16, Lemma 2]. (A more comprehensive generalisation is described in Remark 4.5 (2) below.)

Lemma 4.4. If $H_{n}(B, \mathbb{Z})$ is finitely generated but $H_{n}(C, \mathbb{Z})$ is not, then $H_{n+1}(\Gamma, \mathbb{Z})$ is not finitely generated for any $H N N$ extension of the form $\Gamma=B{ }^{*} C$.

Proof. The Mayer-Vietoris sequence for the HNN extension contains an exact sequence

$$
H_{n+1}(\Gamma, \mathbb{Z}) \rightarrow H_{n}(C, \mathbb{Z}) \rightarrow H_{n}(B, \mathbb{Z}) .
$$

Proof of Theorem 4.1 We shall derive a contradiction from the assumption that $H_{3}(\Gamma, \mathbb{Z})$ is finitely generated for all subgroups of finite index $\Gamma<\mathfrak{X}(F)$.

As in the proof of Lemma 2.1, there is a subgroup of finite index $F_{0}<F$ such that $\Gamma:=L \rtimes F_{0}$, which has finite index in $\mathfrak{X}(F)$, is an HNN extension $B \star_{L}$ with base group $B:=L \rtimes F_{1}$ and associated subgroup $L=L(F)$; the stable letter commutes with $L$.

$\Gamma$ is finitely presented and $H_{3}(\Gamma, \mathbb{Z})$ is finitely generated. $B$, as a retract of $\Gamma$, inherits these properties. By Lemma 4.4 , this implies that $H_{2}(L, \mathbb{Z})$ is finitely generated. By applying Lemma 4.3 to the short exact sequence $1 \rightarrow W \rightarrow L \rightarrow \rho(L) \rightarrow 1$, noting that $\rho(L)<F \times F$ has cohomological dimension 2 , we deduce that $W$ is finitely generated. This contradicts Lemma 4.2. so the proof is complete.

Remarks 4.5. (1) Care is needed in interpreting the logic of the above proof: we did not prove that $W(F)$ and $H_{2}(L(F), \mathbb{Z})$ are not finitely generated.

(2) The more comprehensive homological version of Miller's lemma alluded to above is the following: Suppose $G$ is the fundamental group of a finite graph of groups where all vertex groups $G_{v}$ are of type $\mathrm{FP}_{m}$; if $G$ is of type $\mathrm{FP}_{m}$, then all of edge groups $G_{e}$ are of type $\mathrm{FP}_{m-1}$. 


\subsection{Related observations.}

Proposition 4.6. Let $G$ be a finitely presented group. Then $W(G)$ is finitely generated if $H_{2}(P, \mathbb{Z})$ is finitely generated, where $P=\left\langle\left(g, g^{-1}\right): g \in G\right\rangle$ is the kernel of the map $G \times G \rightarrow G / G^{\prime}$ that restricts to the natural surjection on each factor.

Proof. Consider the LHS spectral sequence in homology for the central extension $1 \rightarrow W \rightarrow L \rightarrow$ $\rho(L) \rightarrow 1$ and note that $\rho(L)=P$, so the spectral sequence $E_{p, q}^{2}=H_{p}\left(P, H_{q}(W, \mathbb{Z})\right)$ converges to $H_{p+q}(L, \mathbb{Z})$. Then $E_{1,0}^{\infty}=E_{1,0}^{2}=H_{1}(P, \mathbb{Z}), E_{0,1}^{\infty}=E_{0,1}^{3}=\operatorname{Coker}\left(d_{2,0}^{2}\right)$, where $d_{2,0}^{2}: E_{2,0}^{2}=$ $H_{2}(P, \mathbb{Z}) \rightarrow E_{0,1}^{2}$, and there is an exact sequence

$$
E_{0,1}^{\infty} \rightarrow H_{1}(L, \mathbb{Z}) \rightarrow E_{1,0}^{\infty}
$$

By a result of Lima and Oliveira 15 (or Proposition 2.3 above) $H_{1}(L, \mathbb{Z})$ is finitely generated. Hence $E_{0,1}^{\infty}=\operatorname{Coker}\left(d_{2,0}^{2}\right)$ is finitely generated, and since $H_{2}(P, \mathbb{Z})$ is finitely generated this implies $E_{0,1}^{2}=H_{0}(P, W)=W$ is finitely generated.

Corollary 4.7. When $H_{2}(P, \mathbb{Z})$ is finitely generated, $L$ is finitely presented (respectively, $\mathrm{FP}_{k}$ ) if and only if $P$ is finitely presented (respectively, $\mathrm{FP}_{k}$ ).

Proof. Proposition 4.6 gives a short exact sequence $1 \rightarrow W \rightarrow L \rightarrow P \rightarrow 1$ and $W$ is a finitely generated abelian group.

From the 1-2-3 Theorem [2], [7] we get the following consequence of Proposition 4.6.

Corollary 4.8. If $G$ is finitely presented and $[G, G]$ is finitely generated, then $W(G)$ is finitely generated and $L(G)$ is finitely presented.

Proof. Under these hypotheses, the 1-2-3 Theorem implies that $P=\rho(L)$ is finitely presented, hence $W$ is a finitely generated abelian group and $L$, which fits into the exact sequence $1 \rightarrow$ $W \rightarrow L \rightarrow P \rightarrow 1$, is finitely presented (and is of type $\mathrm{FP}_{k}$ if and only if $P$ is).

Remarks 4.9. (1) In [13] Kochloukova and Sidki showed that when the abelianization of $[G, G]$ is finitely generated then $W(G)$ is finitely generated.

(2) The converse of Proposition 4.6 is not true. For example, if $G=\left\langle a, t \mid t^{-1} a t=a^{2}\right\rangle$ then $H_{2}(P, \mathbb{Z})$ is not finitely generated ([1], Example 2) but Kochloukova and Sidki [13] prove that $W(G)=0$.

\section{More on the StruCture of $W(G)$}

Let $v_{m}$, as in (1.5), be the number of relators $[w, \bar{w}]$ required to present $\mathfrak{X}\left(F_{m}\right)$.

Lemma 5.1. If $G$ is m-generator, i.e. $d(G)=m$, then $d\left(H_{2}(\mathfrak{X}(G), \mathbb{Z})\right) \leq 2 d\left(H_{2}(G, \mathbb{Z})\right)+v_{m}$.

Proof. Let $N$ be the kernel of the natural map $G * \bar{G} \rightarrow \mathfrak{X}(G)$; it is the normal closure of $v_{m}$ commutators and therefore $H_{0}\left(\mathfrak{X}(G), H_{1}(N, \mathbb{Z})\right)$ requires at most $v_{m}$ generators. From the 5 -term exact sequence in homology associated to $G * \bar{G} \rightarrow \mathfrak{X}(G)$ we get an exact sequence

$$
H_{2}(G * \bar{G}, \mathbb{Z}) \rightarrow H_{2}(\mathfrak{X}(G), \mathbb{Z}) \rightarrow H_{0}\left(\mathfrak{X}(G), H_{1}(N, \mathbb{Z})\right) \rightarrow H_{1}(G * \bar{G}, \mathbb{Z}) .
$$

And $H_{2}(G * \bar{G}, \mathbb{Z}) \cong H_{2}(G, \mathbb{Z}) \oplus H_{2}(G, \mathbb{Z})$.

Proposition 5.2. Let $G$ be a perfect m-generator group. If $\left.d\left(H_{2}(G), \mathbb{Z}\right)\right)>v_{m}$, then $W(G)$ is non-trivial.

Proof. As $G$ is perfect, $\rho(G)=G \times G \times G$ and $H_{2}(\rho(G), \mathbb{Z}) \cong H_{2}(G, \mathbb{Z}) \times H_{2}(G, \mathbb{Z}) \times H_{2}(G, \mathbb{Z})$. From the 5 -term exact sequence for $1 \rightarrow W \rightarrow \mathfrak{X}(G) \rightarrow \rho(G) \rightarrow 1$ we get an exact sequence

$$
H_{2}(\mathfrak{X}(G), \mathbb{Z}) \rightarrow H_{2}(\rho(G), \mathbb{Z}) \rightarrow H_{0}(\mathfrak{X}(G), W),
$$

so if $H_{0}(\mathfrak{X}(G), W)=0$ then $d\left(H_{2}(\mathfrak{X}(G), \mathbb{Z})\right) \geq d\left(H_{2}(\rho(G), \mathbb{Z})\right)=3 d\left(H_{2}(G), \mathbb{Z}\right)$, contradicting Lemma 5.1

Question 5.3. For $F$ a finitely generated free group, what is $W(F)$ ? Does $\mathfrak{X}(F)$ have finite cohomological dimension? 
We shall resolve this question in a future paper [9]. In particular we prove that if the rank of $F$ is at least 3, then $W(F)$ is not finitely generated.

\section{REFERENCES}

[1] Baumslag G., Bridson M. R., Holt D. F., Miller C. F. Finite presentation of fibre products of metabelian groups, J. Pure Appl. Algebra 181 (1) (2003) 15 - 22.

[2] Baumslag G., Bridson M.R., Miller C.F., Short H., Fibre products, non-positive curvature, and decision problems, Comm. Math. Helv. 75 (2000), 457-477.

[3] Bestvina M., Brady N., Morse theory and finiteness properties of groups, Invent. Math. 129 (1997) 445470.

[4] Bieri R., Homological dimensions of descrete groups, Mathematics Dept., Queen Mary College, University of London, 1981

[5] Bieri R., Strebel R., Almost finitely presented soluble groups, Comment. Math. Helv. 53 (1978), no. 2, 258 278

[6] Bridson M. R., Howie J., Miller C.F., Short H., Subgroups of direct products of limit groups, Ann. of Math. (2) 170 (2009), no. 3, 1447 - 1467

[7] Bridson M.R., Howie J., Miller C.F., Short H., On the finite presentation of subdirect products and the nature of residually free groups, Amer. J. Math., 135 (2013), 891 - 933.

[8] Bridson M.R., Kochloukova D.H., Weak commutativity, virtually nilpotent groups and Dehn functions, in preparation.

[9] Bridson M.R., Kochloukova D.H., Preprint in preparation.

[10] Bridson M.R., Miller C.F., Structure and finiteness properties of subdirect products of groups, Proc. Lond. Math. Soc. (3) 98 (2009), 631 - 651.

[11] Gupta N., Rocco N., Sidki S., Diagonal embeddings of nilpotent groups, Illinois J. Math. 30 (1986), no. 2, $274-283$

[12] Hall M. Jr., Subgroups of finite index in free groups, Canad. J. Math. 1 (1949), 187 - 190

[13] Kochloukova D., Sidki, S., Weak commutativity in groups, J. Algebra (2017), 471, 319 - 347

[14] Leary I. J., Uncountably many groups of type FP, arXiv:1512.06609

[15] Lima B. C. R., Oliveira R. N., Weak commutativity between two isomorphic polycyclic groups, J. Group Theory (2016), Vol 19, Iss. 2, 239 - 248

[16] Miller C.F., The second homology group of a group; relations among commutators, Proc. Amer. Math. Soc. 3 (1952), $588-595$

[17] Serre J. P., Cohomologie des groupes discrets, Ann. of Math. Studies 70 (1971), 77 - 169.

[18] Rocco N. R., On weak commutativity between finite p-groups, p odd, J. Algebra 76 (1982), no. 2, 471 - 488

[19] Sidki S., On weak permutability between groups, J. Algebra 63 (1980), no. 1, 186 - 225.

[20] Stallings J. R., A finitely presented group whose 3-dimensional homology group is not finitely generated, Amer. J. Math. , 85, (1963) 541 - 543.

[21] Wall C. T. C., Finiteness conditions for CW-complexes, Ann. of Math. (2) 81 (1965), 56 - 69.

Mathematical Institute, University of Oxford, Andrew Wiles Building, ROQ, Oxford OX2 6GG, United KINGDOM

E-mail address: bridson@maths.ox.ax.uk

Department of Mathematics, State University of Campinas (UniCAMP), 13083-859, Campinas, SP, BRAZIL

E-mail address: desi@ime.unicamp.br 